lesions play a role by disruption of crucial tracts, but the effect of T2-visible lesions on MS-related cognitive impairment is limited. Detection of cortical lesions in critical brain areas is important, and cerebral atrophy is robustly associated with cognitive deficits. Brain volume measures are correlated better with cognitive performance than T2 and T1 lesion volumes. The value of fMRI in measurement of benefits of therapeutic interventions in MS requires multicenter studies. (Filippi M, Rocca MA, Benedict RHB, et al. The contribution of MRI in assessing cognitive impairment in multiple sclerosis. Neurology Dec 7, 2010;75:2121-2128). (Response and Reprints: Dr Massimo Filippi. Email: m.fillipi@hsr.it).

COMMENT. Cognitive impairment occurs in $40 \%$ to $70 \%$ of patients with MS. Deficits involve chiefly information processing speed and episodic memory (repetition or recall of verbal or visual information presented over successive learning trials, after an interval of 20-30 minutes). Two validated test batteries are accepted: the Rao Brief Repeatable Neurosychological Battery (BRNB) and the Minimal Assessment of Cognitive Function in Multiple Sclerosis (MACFIMS). The Symbol Digit Modalities Test (SDMT) requires only 5 minutes and is proposed as a reliable and sensitive test in MS, correlating with MRI findings.

\title{
NEUROCOGNITIVE SEQUELAE IN AFRICAN AMERICAN AND CAUCASIAN CHILDREN WITH MS
}

Psychologists at the University of Alabama, Birmingham, examined neurocognitive differences between African American (AA) and Caucasian (CA) patients with pediatric-onset multiple sclerosis (POMS). The study included 42 subjects aged 6-21 years; $20 \mathrm{AA}$ and $22 \mathrm{CA}$. The AA cohort performed worse on measures of language $(\mathrm{p}<0.001)$ and complex attention $(\mathrm{p}<0.01)$ than their CA peers. Longitudinal measurement of cognitive pathology is important for development of effective intervention strategies to prolong cognitive functioning in POMS patients. (Ross KA, Schwebel DC, Rinker J II, et al. Neurocognitive sequelae in African American and Caucasian children with multiple sclerosis. Neurology Dec 7, 2010;75:2097-2102). (Response and Reprints: Kelly Ross MA, Dept Psychology, University of Alabama, 1530 $3^{\text {rd }}$ Ave South, CH 415, Birmingham, AI, 35294 . F-mail: kross3@uab edu!).

COMMENT. The authors suggest that cognitive intervention focused on language and complex attention skills may be particularly helpful in AA youth with MS.

In an editorial, Marie RA (Neurology 2010;75:2054-2055) comments that heterogeneity in physical and cognitive outcomes in MS reflect the contribution of multiple factors including genetics, environment, comorbid disease, health behavior, and increasingly, race and ethnicity. Ethnicity, or a social group who share a common history, social and cultural traditions, may be underappreciated in evaluating differences in cognitive ability. Longitudinal studies in children with MS need to measure differences related to ethnicity as well as race and social factors. 